International Journal of Computer Science, Engineering and Applications (IJCSEA) Vol.2, No.1, February 2012

\title{
ENERGY COMPUTATION FOR BCI USING DCT AND MOVING AVERAGE WINDOW FOR NOISE SMOOTHENING
}

\author{
Ch.Aparna ${ }^{1}$, Dr. J.V.R.Murthy ${ }^{2}$,Dr. B.Raveendra Babu ${ }^{3}$ \\ ${ }^{1}$ R.V.R\&J.C College of Engineering, Guntur, A.P. \\ chaparala_aparna@yahoo.com \\ ${ }^{2}$ Dept. CSE, JNTU College of Engineering, Kakinada, A.P. \\ mjonnalagedda@gmail. com
}

${ }^{3}$ DELTA Technology \& Management Services Pvt. Ltd., Madhapur, Hyderabad, A.P.

rbhogapathi@yahoo.com

\begin{abstract}
Brain computer interface (BCI) is a fast evolving field of research enabling computers and machines to be directly controlled by the human neural system. This enables people with muscular disability to directly control machines using their thought process. The brain signals are recorded using Electroencephalography (EEG) and patterns extracted so that the BCI system should be able to classify various patterns of brain signal accurately to perform different tasks. The raw EEG signal contains different kinds of interference waveforms (artifacts) and noise. Thus raw signals cannot be directly used for classification, the EEG signals has to undergo preprocessing, to remove artifacts and to extract the right attributes for classification. In this paper it is proposed to extract the energies in the EEG signal and classify the signal using Nä̈ve Bayes and Instance based learners. The proposed method performs well for the two class problem in the multiple datasets used..
\end{abstract}

\section{KEYWORDS}

BCI, EEG, Butterworth filters, Moving average spencer window, Discrete cosine transform (DCT), IBL.

\section{INTRODUCTION}

A Brain computer interface (BCI) system works by recording the brain signals and applying machine learning algorithms to classify the brain signals and performing a computer controlled action. BCI finds application for physically impaired people as no peripheral muscular activity is required. The most widely used method for signal acquisition is the electro-encephalography (EEG). The reason for the popularity of EEG is due to the non-invasive way of acquiring the brain signals and also it is safe, easy and cheap when compared to other methods. Most of the existing application prototypes of BCI use EEG signals. Prototypes like "Thought Translation Device" [1] which allows paralyzed patient to write sentences, spelling system [2], "virtual keyboard" based on motor activity [3] are all EEG based BCI systems. Thus the role of EEG processing is crucial in the development of BCI.

DOI : 10.5121/ijcsea.2012.2102 
The user can control BCI by producing different brain activity patterns which is identified by the system and translated into commands. BCI automatically estimates the class of data using classification algorithm. Investigation and evaluation of different classification algorithms for EEG-based BCI is available in literature [4, 5]. Translating brain activity into a computer command is achieved using Regression [6] or classification [7] algorithms.

Bassani et al.,(2010) [8] proposed continuous wavelet transformation (CWT) and wavelet coherence (WC) for feature extraction. Patterns were extracted in the time-frequency plane and optimized. Using WC analysis, the CWT allows the representation of time-frequency patterns of the signal's information content. The proposed feature extraction method was tested using the Naive Bayes classifier with good results.

Rutkowski et al.,(2009)[9] extensively investigated to remove artefacts caused by muscular interference due to eye movement during the EEG signal recording. The proposed method used correlation of the oscillatory modes within multi channel signal in the Hilbert domain.The proposed method was examined and found to decrease the unwanted noise introduced during involuntary movement of the eye.

Lotte et al.,(2009) [10] use fuzzy logic to obtain the region of interest. The proposed model, Fuzzy Region of Interest Activity (FuRIA) is based on inverse solutions and new concepts of fuzzy region. The advantage of the proposed algorithm is its trainability and the ROI and fuzzy frequency bank were able to distinguish different mental states. The proposed technique was compared with two data sets from the BCI competitions. The evaluations assessed the impact of hyper-parameters and the effects of fuzzification on the performance. The feature extracted using FURIA was tested using Support Vector Machine (SVM) classifier.

In this paper it is proposed to extract maximum and average energy in the $5-30 \mathrm{~Hz}$. To extract the signals in the $5-30 \mathrm{~Hz}$ range and remove random noise and at the same time maintaining a sharp step response we use two stages of filtering with the first stage being a butterworth filter and the second stage consisting of an moving average 7 point spencer filter. The obtained features were used as classification attributes for Naïve Bayes and Instance Based Learners(IBL) algorithm.

\section{MATERIALS AND METHODS}

Filters allow a specified frequency range to pass through while attenuating the specified unwanted frequency range. Low-pass filter passes low-frequency signals reduces the amplitude of signals with frequencies higher than the cut-off frequency. High-pass filter passes high frequencies and attenuates frequencies lower than its cut-off frequency. A band-pass filter is a combination of a low-pass and a high-pass filter; it passes a certain band of frequencies.

The Butterworth filter is a signal processing filter which is so designed that the output is as flat a frequency response possible in the passband. The Butterworth filter is also called maximally flat magnitude filter. The frequency response of the Butterworth filter has no ripples in the passband and rolls off towards zero in the stopband [11]. It has slower roll-off and more linear phase response when compared to other filters like Chebyshev and elliptic filter. Butterworth filters are advantageously used to filter EEG signals as the pass- and stop-band are maximally flat, which results in quality output signal for different frequency band.

The low-pass filter can be modified into high-pass filter; when placed in series with others to form band-pass and band-stop filters. The gain $G(\omega)$ of an n-order Butterworth low pass filter [12] in terms of transfer function $\mathrm{H}(\mathrm{s})$ is 
International Journal of Computer Science, Engineering and Applications (IJCSEA) Vol.2, No.1, February 2012

$$
G^{2}(\omega)=|H(j \omega)|^{2}=\frac{G_{0}^{2}}{1+\left(\frac{\omega}{\omega_{c}}\right)^{2 n}}
$$

Where $\mathrm{n}$ is order of filter, $\omega_{\mathrm{c}}$ is cut-off frequency and $\mathrm{G}_{0}$ is the DC gain i.e. gain at zero frequency.

The Butterworth filter is used to pre-process the EEG signal to remove low and high frequency artefacts. The EEG signals are filtered with a second order Butterworth filter with cut-off frequencies in a range of $5-30 \mathrm{~Hz}$. In the second stage of filtering a moving average window with Spencer's 7 point is utilized. The moving average window [13] is weighted and given by

$$
y_{t}=\sum_{r=0}^{q} a_{r} X_{r}(n+r)
$$

Where $a_{r}$ is a set of weights, $X_{t}$ is the input time series

The symmetric weights for the spencer's 7 point moving average window is given by $[-0.05874$ $\left.\begin{array}{llllll}0.05874 & 0.29371 & 0.41257 & 0.29371 & 0.05874 & -0.05874\end{array}\right]$.

Once the filtering is done the maximum and the average energy is extracted in the frequency domain using the Discrete Cosine Transform (DCT) [14]. DCT is a transformation method for converting a time series signal into basic frequency components. Some simple functions to compute the DCT and to pre-process the provided EEG data for BCI system are as follows:

The discrete cosine transform of a list of $\mathrm{n}$ real numbers $\mathrm{s}(\mathrm{x}), \mathrm{x}=0, \ldots, \mathrm{n}-1$, is the list of length $\mathrm{n}$ given by:

$$
S(u)=\sqrt{2 / n} C(u) \sum_{x=0}^{n-1} s(x) \cos \frac{(2 x+1) u \pi}{2 n} \quad \mathrm{u}=0, \ldots, \mathrm{n}
$$

Where $\mathrm{C}(\mathrm{u})$ is equal to $1 /$ square root of 2 for $\mathrm{u}=0$ or is equal to 1 for all other values. Each element of the transformed list $\mathrm{S}(\mathrm{u})$ is the inner product of the input list $\mathrm{s}(\mathrm{x})$ and a basis vector. The constant factors are chosen so that the basis vectors are orthogonal and normalized.

The list $\mathrm{s}(\mathrm{x})$ can be recovered from its transform $\mathrm{S}(\mathrm{u})$ by applying the inverse cosine transform (IDCT):

$$
s(x)=\sqrt{2 / n} \sum_{u=0}^{n-1} C(u) S(u) \cos \frac{(2 x+1) u \pi}{2 n} \quad \mathrm{x}=0, \ldots, \mathrm{n}
$$

This equation expresses $\mathrm{s}$ as a linear combination of the basis vectors. The coefficients are the elements of the transform $\mathrm{S}$, which may be regarded as reflecting the amount of each frequency present in the input $\mathrm{s}$. Energies are obtained for all the channels individually using the above said method.

The Naïve Bayes is a probabilistic method used for prediction[14]. During training the conditional probabilities of each attribute in the predicted class is estimated from the training data set. The parameter's mean and variance obtained from a small set of training data is sufficient for 
classification. Commonly used as the Naïve Bayes provide good results and easy interpretation of the results[15,16].

The disadvantage being that the classifier considers that the occurrence of attributes is independent; therefore the correlation between the attributes is ignored.

Instance based learner uses the Euclidean distance based algorithm to obtain the class of the test instances[17]. Instance based learners use a weighted overlap of the feature values of test instance and a memorized example. Metrics used in Instance based learners combine a per feature distance metric with global feature weights that account for relative differences in discriminative power of the features.

In this work we use two datasets available publicly to validate the proposed methodology. The first dataset is obtained from experiments jointly conducted by University of Tübingen, Germany, Dept. of Computer Engineering and Institute of Medical Psychology and Behavioural Neurobiology, and Max-Planck-Institute for Biological Cybernetics, Tübingen, Germany, and Universität Bonn, Germany, Dept. of Epileptology. In the experiment conducted the subject imagines the movement of left small finger or tongue. The time series of the electrical brain activity was picked up using 64 electrodes placed in the contralateral motor cortex. The second data set is provided by Fraunhofer FIRST, Intelligent Data Analysis Group, and University Medicine Berlin, Department of Neurology. In the second experiment 118 electrodes were used with motor imageries of left hand and right foot. Figure I and figure II shows the time domain signal of some electrodes during the motor activity.

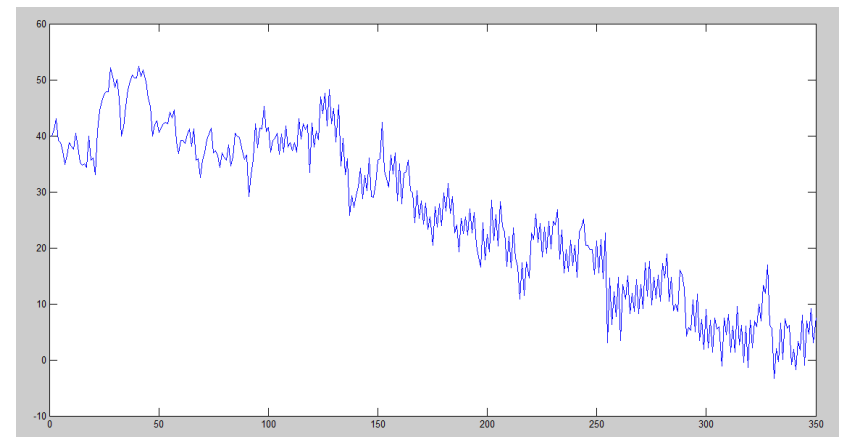

Figure I : 3.5 second epoch from one of the electrodes

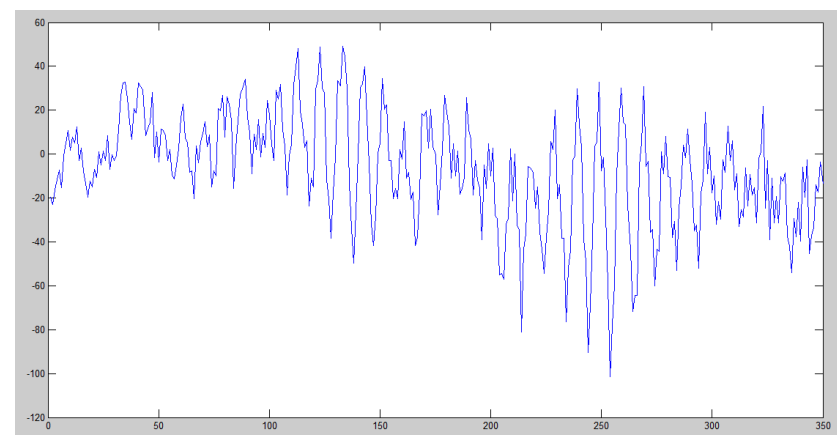

Figure II : 3.5 second signal during imagination of hand movement. 
International Journal of Computer Science, Engineering and Applications (IJCSEA) Vol.2, No.1, February 2012

Figure III shows the output in the frequency domain of the input time domain data.

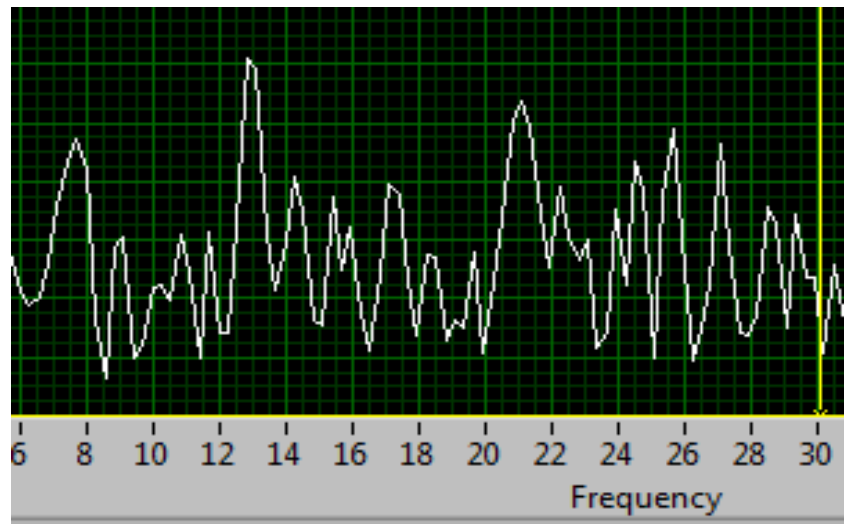

Figure III. The processed signal for feature extraction in frequency domain.

\section{RESULT AND DISCUSSION}

The classification accuracy using Naïve Bayes(NB) and Instance Based Learners(IBL) using the proposed method is shown in figure IV and the relative absolute error is shown in figure $\mathrm{V}$.

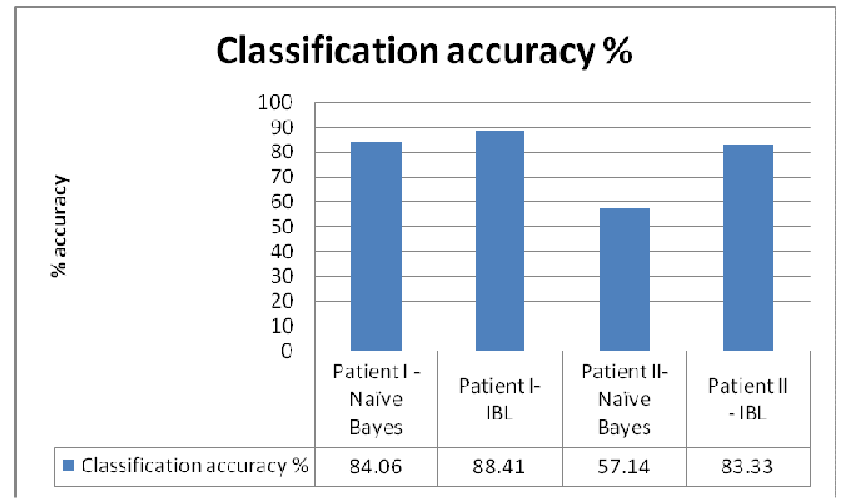

Figure IV : Classification accuracy of NB and IBL for the two dataset.

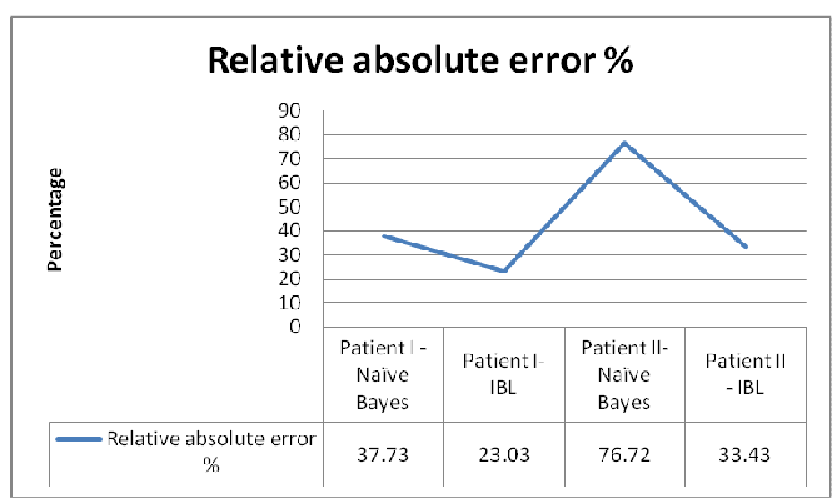

Figure V : Relative absolute error. 
From figure IV and V it can be seen that Instance based learners are more consistent across different datasets. Also being a simple mechanism for computing the class, IBL is proved to be efficient and fast method for BCI.

\section{CONCLUSION}

In this paper the Brain Computer Interface classification problem was investigated. It was proposed to extract maximum and average energy of EEG features using Discrete cosine transform. As the frequency of interest is broadly in the region of alpha and beta waves, Butterworth band pass filter was used to separate the frequency of interest. Moving average Spencer window was used to remove noise among adjacent data values as EEG data is highly noisy. Using 10 fold cross, the proposed feature extraction method was trained and tested using Naïve Bayes classifier and Instance based learner. Two publicly available datasets were used to test the proposed methodology. It is found that Instance based classifier provides consistent results across different EEG datasets. Future work in the direction of using Neural Network classifiers need to be investigated for improving the classification accuracy as the EEG data is highly susceptible to noise.

\section{REFERENCES}

[1] N. Birbaumer, A. Kubler, N. Ghanayim, T. Hinterberger, J. Perelmouter, J. Kaiser, I. Iversen, B. Kotchoubey, N. Neumann, and H. Flor. The Thought Translation Device (TTD) for completely paralyzed patients. In IEEE Transactions on Rehabilitation Engineering, volume 8, pages 190-193, 2000.

[2] E. Donchin, K.M. Spencer, and R. Wijensinghe. The mental prosthesis: Assessing the speed of a P300-based brain-computer interface. In IEEE Transactions on Rehabilitation Engineering, volume 8, pages 174-179, 2000.

[3] R. Scherer, G.R. M“uller, C. Neuper, B. Graimann, and G. Pfurtscheller. An asynchronously controlled EEG-based virtual keyboard: improvement of the spelling rate. In IEEE Transactions on Biomedical Engineering, volume 51(6), pages 979-984, 2004.

[4] D. J. McFarland, C. W. Anderson, K.-R. Muller, A. Schlogl, and D. J. Krusienski. Bci meeting 2005workshop on bci signal processing: feature extraction and translation. IEEE Transactions on Neural Systems and Rehabilitation Engineering, 14(2):135 \{ 138, 2006.

[5] R. O. Duda, P. E. Hart, and D. G. Stork. Pattern Recognition, second edition. WILEYINTERSCIENCE,2001.

[6] D. J. McFarland and J. R. Wolpaw. Sensorimotor rhythm-based brain computer interface (bci): feature selection by regression improves performance. IEEE Transactions on Neural Systems and Rehabilitation Engineering, 13(3):372-379, 2005.

[7] W. D. Penny, S. J. Roberts, E. A. Curran, and M. J. Stokes. Eeg-based communication: a pattern recognition approach. IEEE Transactions on Rehabilitation Engeneering, 8(2):214-215, 2000.

[8] Bassani, T. and Nievola, J.C. "Brain-computer interface using wavelet transformation and naïve Bayes classifier",Advances in Experimental Medicine and Biology, Vol. 657, No. 2 , pp. 147$165,2010$.

[9] Rutkowski, T.M., Cichocki, A., Tanaka, T, Mandic, D.P., Jianting Cao, and Ralescu, A.L. “ Multichannel spectral pattern separation - An EEG processing application”, IEEE International Conference on Acoustics, Speech and Signal Processing, Washington, DC, USA, 2009.

[10] Lotte, F., Lecuyer, A. and Arnaldi, B. "FuRIA: An Inverse Solution Based Feature Extraction Algorithm Using Fuzzy Set Theory for Brain-Computer Interfaces”, IEEE Transactions on Signal Processing, Vol.57, No.8, pp.3253-3263, 2009.

[11] Giovanni Bianchi and Roberto Sorrentino (2007). Electronic filter simulation \& design. McGraw-Hill Professional. pp. 17-20. ISBN 9780071494670.

[12] In Wireless Engineer (also called Experimental Wireless and the Wireless Engineer), vol. 7, 1930, pp. 536-541 - "On the Theory of Filter Amplifiers"-S. Butterworth 
International Journal of Computer Science, Engineering and Applications (IJCSEA) Vol.2, No.1, February 2012

[13] Cadzow.J.A,Wilkes.D.M, Peters.R.A,Li.X.Image texture synthesis-by-analysis using moving-average models. IEEE Transactions on Aerospace and Electronic Systems, Volume: 29 , Issue: 4, pp: 11101122, 1993

[14] Singer.B, Veloso.M.M.Automating the modeling and optimization of the performance of signal transforms. IEEE Transactions on Signal Processing, Volume : 50, Issue:8, pp: 2003-2014. 2002.

[15] Besserve.M,Garnero.L,Martinerie.J.Cross-Spectral Discriminant Analysis (CSDA) for the classification of Brain Computer Interfaces. 3rd International IEEE/EMBS Conference on Neural Engineering, 2007. CNE '07. pp:375 - 378,2007

[16] Coyle. D, McGinnity.T.M,Prasad.G.Identifying Local Ultrametricity of EEG Time Series for Feature Extraction in a Brain-Computer Interface.29th Annual International Conference of the IEEE Engineering in Medicine and Biology Society, 2007. pp:701-704.

[17] Cufoglu.A,Lohi.M,Madani.K.Classification accuracy performance of Naïve Bayesian (NB), Bayesian Networks (BN), Lazy Learning of Bayesian Rules (LBR) and Instance-Based Learner (IB1) comparative study. International Conference on Computer Engineering \& Systems, 2008. pp:210215.

\section{Authors}

Aparna Chaparala is working as an Associate Professor of the department of computer science and engineering in R.V.R. \& J.C. Collegeof Engineering, Chowdavaram,Guntur. She has 10 years of teaching experience. She completed her M.Tech in Computer Sicence \& Engineering. She is doing her research in the area of Data Mining. Presently pursuing Ph.D from J.N.T.U, Hyderabad. She has published 4 papers in international journals.

Dr J.V.R. Murthy is presently working as a professor in the department of CSE at J.N.T.U., Kakinada. He is awarded Ph.D. in CSE from JNTU. He did M.Tech in CSE at IIT. He has 20 years of teaching experience and 3 years industrial experience. A Memento of Appreciation was awarded for "good performance and on schedule completion of People Soft HRMS project" by Key Span Energy Corporation, New York. He has more than 15 publications in national and international journals. His interested areas of research include Data Warehousing, data mining and VLDB.

Dr B. Raveendra Babu has obtained Masters degree in Computer Science and Engineering from Anna University, Chennai. He received Ph.D. in Applied Mathematics from S.V University, Tirupati. He is currently leading a Team as Director (Operations), M/s.Delta Technologies (P) Ltd.,Madhapur, Hyderabad. He has 26 years of teaching experience. He has more than 25 international \& national publications to his credit. His interested areas of research include VLDB, Image Processing, Pattern analysis and Wavelets 